\title{
Plans to Increase Source Brightness of NSLS X-Ray Ring*
}

\author{
J. Safranek and S. Krinsky \\ National Synchrotron Light Source \\ Brookhaven National Laboratory \\ Upton, NY 11973
}

\section{Abstract}

We discuss plans to increase the NSLS X-Ray ring source brightness by an order of magnitude. Proposed improvements include doubling current from $250 \mathrm{~mA}$ to $500 \mathrm{~mA}$, reducing vertical emittance by a factor of 6 and reducing insertion device gaps and periods by up to a factor of two. Experimental results are reported which indicate we have succeeded in reducing the vertical emittance below $2 \dot{A}$.

\section{Introduction}

The NSLS X Ray ring provides a high brightness source of $\mathrm{x}$-rays from bending magnets and insertion devices. In this note we discuss plans to increase the source brightness by an order of magnitude. The recent installation of a fourth $52 \mathrm{MHz}$ RF cavity provides sufficient RF power to allow the stored current to be increased from $250 \mathrm{~mA}$ to $500 \mathrm{~mA}$ [1]. At present, $500 \mathrm{~mA}$ has been stored at injection energy $(750 \mathrm{MeV})$ and $410 \mathrm{~mA}$ at $2.5 \mathrm{GeV}$. However, before running operations at $500 \mathrm{~mA}$, it will be necessary to provide increased cooling downstream of the high power wigglers. A further increase in brightness can be achieved by replacing the existing wigglers by new devices operating with shorter periods and smaller gaps. An experiment [2] is in preparation using the X13 straight section to elucidate the operational limit of the vertical electron beam aperture. Finally, we have embarked on a study aimed at reducing the vertical emittance of the ring, and we have succeeded in reducing the emittance coupling from $1 \%$ to below $0.2 \%$. The remainder of this paper will be devoted to a discussion of our approach to reducing the vertical emittance.

\section{Method of Reducing Vertical Beam Size}

Betatron oscillations in the vertical plane of an electron storage ring can be excited in two ways - horizontal oscillations can be coupled into the vertical plane, or vertical

*Work performed under the auspices of the U.S. Department of Energy uscillations can be excited directly when a photon is radiated where there is nonzero vertical dispersion, $\eta_{y}$. In order to reduce the vertical bcam sizc, $\sigma_{y}$, one must correct both coupling and vertical dispersion. Measurements in the NSLS X-Ray ring indicate that $\eta_{y}$ and coupling give approximately equal contributions to $\sigma_{y}$.

When we started our work to reduce $\sigma_{y}$, the X-Ray ring had eight skew quadrupoles grouped in two families with a single power supply powering the four skews in each fam ily. There was no method for correcting $\eta_{y}$. Now each of the eight original skew quadrupoles have individual power supplies, and nine additional skew quadrupoles have been added. Four of the new skews are located in positions of high $\eta_{x}$, so they can be used to correct $\eta_{y}$ efficiently without exciting much coupling.

CoUPLING: In the past the coupling on the X-Ray ring was minimized by adjusting the two families of skew quadrupoles to minimize the split between the transverse tunes at the difference resonance. With 17 individually powered skew quadrupoles, we needed a better method for measuring coupling than the tune split at the difference resonance. One such method has been developed at Cornell [3] in which the two transverse normal modes are excited on resonance, and the ellipse traced out by the betatron oscillations is measured at the beam position monitors. This method requires turn-by-turn beam position measurement capability which is not available with the present X-Ray ring hardware, so we developed another coupling correction algorithm that takes advantage of the high accuracy closed orbit monitors [4] in the X-Ray ring

We sample the coupling by measuring the shifts in the vertical orbit, $\Delta y$, produced by varying the strengths, individually, of a set of horizontal steering magnets. Then we correct the coupling by determining the skew quadrupole strengths that minimize the vertical orbit shifts. We chose to simultaneously minimize 16 different vertical orbit shifts created by 16 different horizontal steering magnets distributed about the X-Ray ring. The vertical orbit distortion from a single steerer does not have all the coupling information. Skew quadrupoles located at positions of zero orbit distortion for that steering magnet do not create any vertical orbit distortion. Two horizontal stecring magnets separated by close to an odd integer multiple of $90^{\circ}$ in hor- 
izontal betatron phase sample all the skew quadrupoles. We chose to use more than two stccrcrs, because there is some vertical orbit shift that is simply due to imperfect rotational alignment of the steering magnets. This shift is not due to coupling and should not be corrected with the skew quadrupoles. We found that the most effective way to solve this problem is to look at the vertical orbit distortions from many horizontal steering magnets. The vertical distortions really caused by rotated quadrupoles are well corrected with the same skew quadrupole distribution for all the steerers, while the vertical orbit distortions from rotated steerers are randomly distributed and cannot be simultaneously corrected with the skews. We added horizontal steering magnets until we found that adding more no longer changed the skew quadrupole distribution derived to best correct the vertical orbit shifts.

Simulations with PATPET [5] confirmed that there is a strong correlation between the vertical orbit shift and the vertical emittance, and that reducing the vertical shift is an effective way to reduce the vertical emittance (figure 1). Both before and after the skew quadıupoles were adjusted to reduce the vertical orbit shifts, the calculated vertical emittance was found to cxhibit the approximate dependence

$$
\epsilon_{y}(\dot{A}) \approx .002<\frac{\Delta y^{2}(\mu \mathrm{m})}{\beta_{y}(\mathrm{~m})}>
$$

where the shift in the vertical closed orbit, $\Delta y$, results from changing a horizontal steering magnet to give a $3 \mathrm{~mm} \mathrm{rms}$ horizontal orbit distortion.

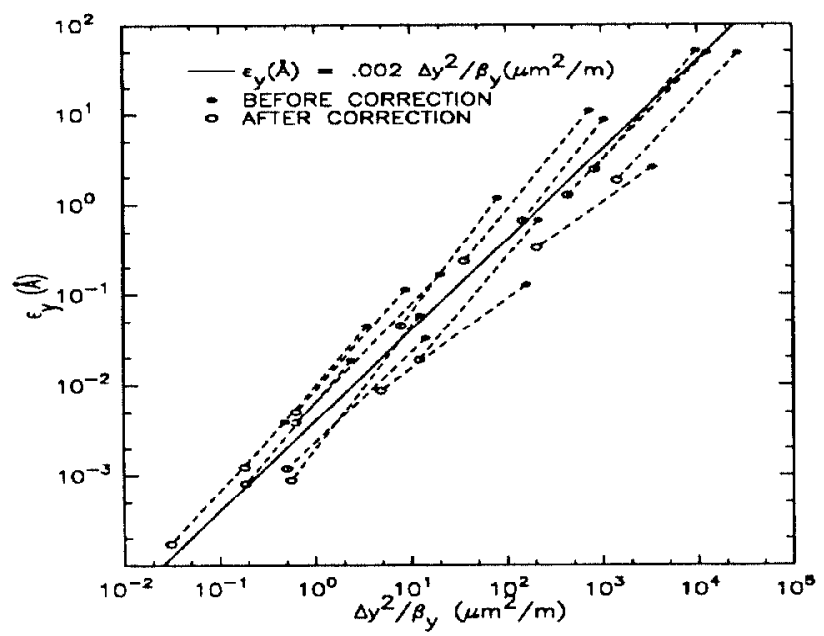

Figure 1: Simulation results from PATPET. Only coupling errors (no $\eta_{y}$ ) were introduced in the lattice. The dotted lines connect points for the same set of quadrupole rotation errors before and after correction.

VERTICAL DISPERSION: In order to minimize quantum excitation and thus minimize the vertical beam size, $\eta_{y}$ should be minimized in the bending magnets where the photons are radiated. In reference [6], the author shows that the emittance from dispersion is given by $\epsilon_{y}=\frac{C_{q} \gamma^{2}}{\rho}<$
$\left.\mathcal{H}_{y}\right\rangle$, where $\left\langle\mathcal{H}_{y}\right\rangle$ is the average value of the CourantSnyder invariant in the bending magnets. In reference [7] the author shows that $\left\langle\mathcal{H}_{y}\right\rangle$ is approximately equal to $2<\frac{\eta_{y}^{2}}{\beta_{y}}>$, so for the X-Ray ring $(\rho=6.9 m$ and $\gamma=5057)$ the vertical emittance from dispersion is given by

$$
\epsilon_{y}(\dot{A}) \approx .03<\frac{\eta_{y}^{2}(\mathrm{~mm})}{\beta_{y}(\mathrm{~m})}>.
$$

Vertical dispersion can be excited by vertical bending or by skew quadrupoles at locations of nonzero $\eta_{x}$. We did not want to change the vertical closed orbit from the standard operational orbit in which the beamlines are steered, so we used skew quadrupoles at locations of large $\eta_{x}$ to correct the vertical dispersion.

Correction Algorithm: The coupling and vertical dispersion correction was done simultaneously by solving a system of linear equations

$$
\mathrm{MK}=\mathrm{V}
$$

using singular value decomposition [8]. Here the vector $\mathbf{V}$ has $48 \times 16+48$ elements. The first $48 \times 16$ elements are the measured vertical orbit shifts, $\Delta y / \sqrt{ } \beta_{y}$, at the 48 beam position monitors for each of 16 horizontal steering magnets. The last 48 elements are the measured, $\eta_{y} / \sqrt{\beta_{y}}$, at the 48 BPMs. The $48 \times 16+48$ by 17 matrix $M$ is the measured change in $\mathbf{V}$ with changes in the 17 skew quadrupoles. The linear equations are solved for $\mathbf{K}$, the 17 skew strengths that minimize the rms of $\Delta y / \sqrt{\beta}_{y}$ and $\eta_{y} / \sqrt{\beta} y$. The relative weight for correcting $\Delta y / \sqrt{\beta}_{y}$ versus $\eta_{y} / \sqrt{\beta} y$ can be adjusted to give more or less correction of coupling versus vertical dispersion.

\section{Experimental Results}

The correction algorithm was very successful in reducing the vertical dispersion and vertical orbit shifts. We were able to reduce $<\frac{\Delta y^{2}}{\beta_{y}}>$ by a factor of nine compared to the value $\left(5500 \mu \mathrm{m}^{2} / \mathrm{m}\right)$ achieved with the previous coupling correcton algorithm using only two families of skew quadrupoles. Figure 2 shows the reduction of $\Delta y / \sqrt{\beta}_{y}$ for a typical one of the sixteen horizontal orbit steering magnets. We were simultaneously able to reduce $\left\langle\frac{\eta_{y}^{2}}{\beta_{y}}\right\rangle$ by a factor of seven (figure 3 ) from a starting value of 300 $\mathrm{mm}^{2} / \mathrm{m}$.

According to equations 1 and 2 , we expect the reduction in $\left\langle\frac{\Delta y^{2}}{\beta_{y}}>\right.$ and $\left\langle\frac{\eta_{y}^{2}}{\beta_{y}}>\right.$ to give a reduction in the vertical emittance of about eight. We measured the emittance reduction in two ways - by measuring the decrease in Touschek lifetime [9] with approximately $80 \mathrm{~m}$ A stored in a single bunch, and by directly measuring the vertical beam size reduction using $x$-ray pinhole cameras. We saw nearly a factor of 2.5 decrease in the Touschek lifetime which indicates about a factor of 6 reduction in the vertical emittance. 


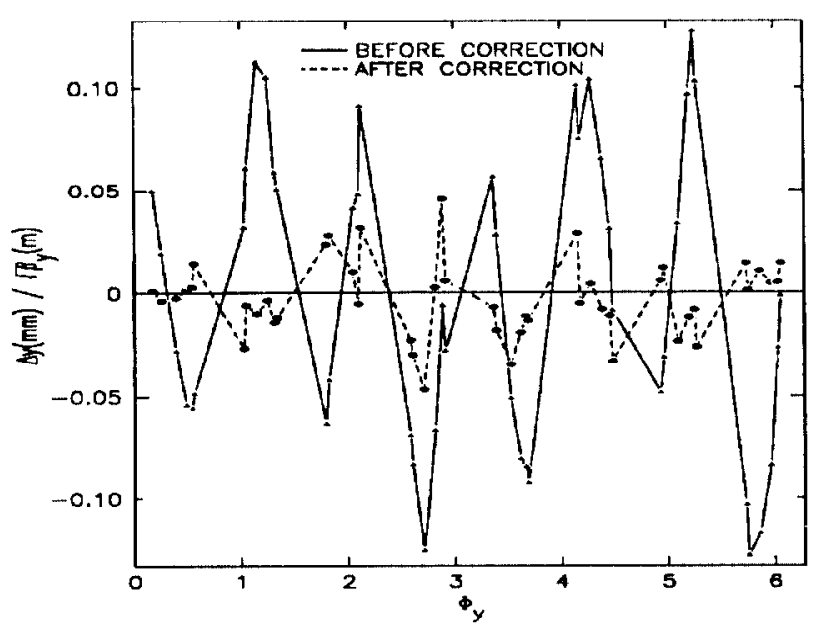

Figure 2: Measured vertical orbit shift resulting from a 3 mm rms horizontal orbit shift from a single steerer.

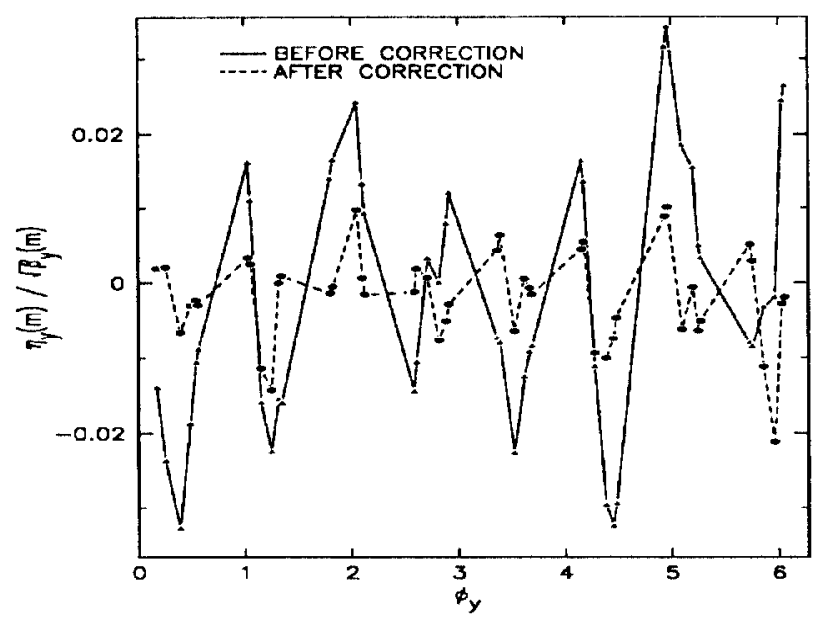

Figure 3: Measured vertical dispersion.

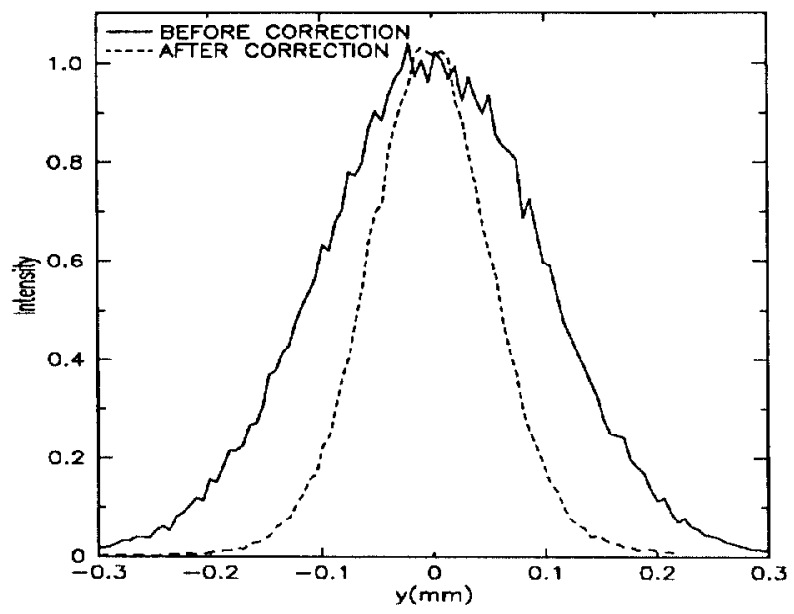

Figure 4: Vertical beam size at $\mathrm{X} 26\left(\beta_{y}=15 \mathrm{~m}\right)$.

We measured the beam size reduction with $\mathrm{x}$-ray pin- hole cameras on beamlines X10 and X26. The results with beamline $\mathrm{X} 26$ appeared to give the best resolution. Figure 4 shows the profile measured at X26 with the old skew quadrupole correction and the smaller profile with the new correction. Subtracting a small resolution factor in quadraturc, these measurements indicate a reduction in electron beam size of 2 which gives a reduction in vertical emittance of 4 .

The measurements on $\mathrm{X} 26$ indicate that the vertical emittance is $6 \dot{A}$ with the old skew quadrupole settings compared to a horizontal emittance of $110 \mathrm{~nm}$. With the new skew correction the X26 measurements indicate a vertical emittance of $1.5 \dot{A}$. The fact that a greater reduction in Touschek lifetime was seen than the reduction in electron beam profile may indicate that we are running into a resolution limit with the X26 pinhole camera. If we assume this is the case and take the true reduction in vertical emittance to be a factor of six as measured with the Touschek lifetime, this would indicate that the vertical emittance with the new skew quadrupole correction is 1 $\dot{A}$. Work is continuing to better understand the resolution and absolute calibration of the pinhole cameras.

\section{Acknowledgements}

We would like to express our sincere appreciation for the help we received measuring the beam size from M. Sansone, S. Sutton, and M. Rivers. Discussions with R. Gluckstern were helpful when we were working to derive a correction algorithm.

\section{References}

[1] M.G. Thomas, R. Biscardi, W. Broome, S. Buda, R. D'Alsace, S. Hanna, J. Keane, P. Mortazavi, G. Ramirez, J.M. Wang, these proceedings.

[2] P.M. Stefan, L. Solomon, S. Krinsky, G. Rakowsky, Procecdings 1991 Part. Accel. Conf. San Francisco, CA, p. 1096.

[3] P. Bagley, D. Rubin, Proceedings 1989 IEEE Part. Accel. Conf. Chicago, IL, p. 874.

[4] R. Biscardi, J.W. Bittner, Proceedings 1989 IEEE Part. Accel. Conf. Chicago, IL, p. 15.

[5] L. Emery, H. Wiedemann, J. Safranek, SSRL/ACD note 36 revised, June, 1988.

[6] M. Sands, SLAC-121, November, 1970.

[7] T. Raubenheimer, SLAC-PUB-4937, February, 1990.

[8] W. Press, B. Flannery, S. Teukolsky, W. Vetterling, Numerical Recipes, Cambridge, 1990.

[9] H. Bruck, Circular Particle Accelerators, Los Alamos report $L A-T R-72-10 \mathrm{rev}$. 